\title{
La práctica reflexiva como estrategia para favorecer la construcción del conocimiento profesional del profesor de biología
}

\author{
Reflexive practicum as a strategy to favor biology teacher's professional knowlegde \\ construction
}

Alice Marcela Gutiérrez $P^{1}{ }^{1}$, Edgar Orlay Valbuena $U^{1}$ y Leidy Marcela Neira A. ${ }^{2}$

${ }^{1}$ Profesores del Departamento de Biología. Universidad Pedagógica Nacional. ${ }^{2}$ Estudiante del Departamento de Biología. Universidad Pedagógica Nacional - Grupo de investigación Conocimiento Profesional del Profesor en Ciencias- galice marcela@hotmail.com, edgarorlay@hotmail.com, margalamar@yahoo.es

\section{Introducción}

"Es la reflexión sobre la experiencia lo que permite que podamos aprender nuevos conocimientos y prácticas, es lo que diferencia una formación experiencial de una formación basada en la impregnación o el mimetismo"

Marcelo (2005)

En el Grupo de Investigación Conocimiento Profesional del Profesor de Ciencias, de la Universidad Pedagógica Nacional, consideramos prioritario asumir en la formación docente (inicial y permanente) como elemento estructurante la Práctica Reflexiva (PR). Al respecto, pensamos que dicha Práctica favorece el reconocimiento que ha de tener el profesor del conocimiento que se construye con relación a la práctica pedagógica del maestro en el aula de clase. Es decir, creemos que en la formación docente no basta con el aprendizaje de los contenidos académicos disciplinares, siendo así necesario sistematizar y reflexionar la praxis.

Contemplando lo anterior, es necesario tener en cuenta que el Conocimiento Profesional (CP) no resulta solo de la relación de los diferentes saberes y conocimientos del profesor, ni mucho menos de la mera identificación de los mismos. El CP demanda un complejo sistema de interrelaciones entre los diversos componentes que la constituyen. Para el caso concreto del Conocimiento Profesional del Profesor de Biología (CPPB), creemos que los distintos componentes se integran alrededor del Conocimiento Didáctico del Contenido Biológico (CDCB).

En esa dirección, el presente taller contiene unas especificidades en cuanto a la aproximación que se quiere llevar a cabo respecto a las prácticas educativas que desarrollan tanto docentes en formación inicial como profesores que en la actualidad se encuentran ejerciendo su profesión en el campo de la enseñanza de la Biología.

\section{Desarrollo}

Para el planteamiento y desarrollo del taller es necesario hacer daridad en cuanto a los fundamentos conceptuales que sustentan la propuesta centrada en la idea de la relevancia de la reflexión en y sobre la práctica docente para identificar los componentes del CPPB, así como de los factores que favorecen su construcción, tomando como elemento estructurador el CDCB (Valbuena, 2007), lo cual se desarrollará a partir de la estrategia de la PR.

Para autores como Porlán y Rivero (1998), la estructuración del Conocimiento Profesional no obedece exdusivamente a la lógica disciplinar, ni a la acumulación de experiencias. Este conocimiento, se construye a partir de las interrelaciones y transformaciones de los diferentes 
saberes (académicos, experienciales, etc), que surgen de los problemas relevantes de la práctica profesional. En palabras de los profesores "es un conocimiento sobre la integración $y$ transformación de saberes en la perspectiva de formular determinado conocimiento escolar y sobre los procesos que facilitan su construcción"(1998: 161). Partiendo de lo planteado por el Proyecto Curricular Innovación y Renovación Escolar - IRES-, es perentorio hacer énfasis en que el conocimiento del profesor de Ciencias no se constituye solamente desde lo que él sabe de una disciplina científica espećfica, siendo prionitario el reconocimiento del acontecer en el contexto escolar lo que le ofrece la oportunidad al maestro de construir su conocimiento profesional.

Desde esta perspectiva consideramos importante ayudarle al profesor a evidenciar que a partir de sus desarrollos, ha ido constituyendo un saber que le es propio y que por ende ha de ser valorado y trabajado desde él mismo; como lo plantea Gutiérrez (2008) cuando establece, las estrategias utilizadas en favor del desarrollo profesional del profesor han de contribuir a que él reconozca que es sujeto de saber, que ha construido un conocimiento con relación a su profesión, que posee unas características particulares que le permiten desarrollar sus propuestas de trabajo y que existe la posibilidad de innovar en el aula de clase desde su práctica.

Otro autor que vemos necesario abordar en el planteamiento de este taller es Maurice Tardif (2004), pues éste establece que el saber profesional tiene un carácter diverso, práctico, temporal, de interacción humana, experiencial, y de interfaz entre lo individual y lo social. En palabras suyas: "El saber no es una cosa que fluctúe en el espacio: el saber de los maestros es el saber de ellos $y$ está relacionado con sus personas y sus identidades, con su experiencia de vida y su historia profesional, con sus relaciones con los alumnos en el aula y con los demás actores escolares del centro, etc. Por eso, es necesario estudiarlo relacionándolo con esos elementos constitutivos del trabajo docente"(2004: 10)

Otra característica que plantea Tardif acerca del saber del profesor lo define a partir del postulado de la subjetividad, en el que establece que los profesores en el contexto de sus tareas cotidianas poseen, utilizan y producen saberes espećficos de su profesión. Es decir, los maestros son actores competentes y sujetos de conocimiento y no simples ejecutores técnicos de los conocimientos producidos por otros (Valbuena, 2007). En términos del autor, el profesor "es un actor en el sentido fuerte de la palabra, es decir, un sujeto que asume su práctica a partir de los significados que él mismo le da, un sujeto que posee conocimientos y un saber hacer provenientes de su propia actividad y a partir de los cuales la estructura y la orienta"(Tardif, 2004: 169).

Retomamos a Tardif como un referente que apoya nuestra propuesta, pues éste ha llevado a cabo sus planteamientos a partir de la investigación con profesores en ejercicio en diferentes aulas de dase, haciendo un reconocimiento de su labor desde los propios docentes. Las investigaciones de este autor apuntan a que los significados y desarrollos que llevan a cabo los profesores no son solamente producto de lo que han estudiado, también es parte de las interpretaciones, reflexiones y elaboraciones que en y sobre la práctica ha tenido que hacer, lo cual fundamenta su desarrollo profesional.

Así pues, para el caso del profesor de Biología, los conocimientos académicos que él posee sobre las diferentes disciplinas, las experiencias, saberes y concepciones sobre la Biología, sobre la enseñanza, el aprendizaje y la educación, son componentes y fuentes a la vez del conocimiento que lo identifica profesionalmente, es decir del CPPB, el aral se construye a partir de la integración y transformación de los primeros (Valbuena, 2007). Según el mismo autor, la práctica docente en la aral se enseña Biología constituye el escenario donde ese conocimiento cobra sentido y donde se construye. 0 sea, es en la dase de Biología donde el profesor se encuentra con dificultades, recursos, situaciones y oportunidades particulares que le llevan a tomar decisiones sobre qué contenidos, qué finalidades y qué actividades preferenciar en la enseñanza. Estamos refiriéndonos al Conocimiento Didáctico del Contenido Biológico, que es el que le permite al profesor realizar la 
transformación didáctica de los diversos saberes y conocimientos para hacer posible la construcción de una Biología que se diferencia de la Biología de los científicos y de los referentes biológicos "del común", nos referimos a la "Biología Escolar". Resumiendo, el CDCB es un componente fundamental en la construcción del CPPB que le permite al profesor estructurar un saber escolar con un estatus epistemológico diferenciado: la "Biología Escolar" (Valbuena, 2007). Pero, dicho conocimiento suele ser más implícito que explícito, con lo aual resulta relevante hacerlo visible con por lo menos dos fines, a nuestra manera de ver: transformar las prácticas docentes propias del profesor de Biología con la consecuente explicitación del CPPB, y constituirse en un objeto de estudio de la Didáctica de la Biología.

Para plantear la PR como una estrategia que favorezca el reconocimiento del profesor sobre sus desarrollos profesionales, nos basaremos en el modelo del Profesional Reflexivo, planteado por Donal Schön (1987). Este autor entiende que la formación eficiente del profesional práctico ha de basarse en su práctica, pues la sitúa en el centro del proceso de aprendizaje, para ligar la teoría a la práctica de forma significativa a posteriori y la dave para conseguir éste vínculo es el pensamiento práctico. Por otra parte, según Perrenoud (2007), una práctica reflexiva supone una postura, una forma de identidad. Su realidad no se considera según el discurso o las intenciones, sino según el lugar, la naturaleza y las consequencias de las reflexiones en el ejercicio cotidiano del oficio, tanto en situación de crisis o de fracaso como a un ritmo normal de trabajo.

Igualmente, el desarrollo de la PR como lo plantea Gutiérrez (2007), ha de favorecer de manera explíaita el reconocimiento por parte del profesor de sus potencialidades personales en cuanto a la construcción de su conocimiento profesional y del cómo ha llegado a los mismos; desde esta perspectiva se posibilitará el desarrollo de atribuciones causales intemas y controlables que a su vez le permitirán autorregularse llevándolo a reconocerse como sujeto de saber que se construye y da sentido a su profesión (Gutiérrez, 2007 p. 159)

Por tal motivo, reconocemos que favorecer el reconocimiento de la PR se convierte en una posibilidad de desarrollo profesional con la que buscamos llevar a los docentes a examinar y valorar el CDCB que han construido a partir de su hacer cotidiano, donde la reflexión sobre la acción los lleve a plantear altemativas que enriquezcan su labor cotidiana partiendo de sus propios procesos de construcción de su labor docente.

\section{PLANTEAMIENTO GENERAL DEL TALLER}

"Se puede hallar sentido en la inmovilidad, la ausencia de decisión o la nutina absoluta. 0 , más exactamente, una vida tranquila y ordenada puede anestesiar la búsqueda de sentido, llevar a no preguntarse nunca por qué hacemos lo que hacemos, con qué derecho, o en virtud de qué sueños"

Perrenoud (2007, p.60)

El objetivo general del taller es abrir un espacio de disausión y reflexión con los profesores sobre la importancia que tiene, en el desarrollo de su Conocimiento Profesional, la Práctica Reflexiva, como medio que lo lleve a reconocer y valorar el conocimiento que ha ido construyendo desde su práctica pedagógica.

Algunas de las características que orientaran el trabajo del taller son:

1. Reconocemos la importancia que tiene el llevar a cabo un constate cuestionamiento, como medio de aproximación tanto a la práctica educativa que lleva el maestro, como también a la reflexión que se puede suscitar por medio de la misma.

2. Abrimos la posibilidad del trabajo individual con la intención de promover la reflexión del maestro sobre su acción profesional, así como otras actividades grupales que potencien la reflexión colectiva con el fin de la aproximación de lo que hacen otros y su práctica pedagógica individual. 
3. Destacamos la necesidad de incentivar a los profesores a que en sus prácticas desarrollen un habitus'con relación a la práctica reflexiva.

4. Organizamos las actividades del taller planteando tres principios generales que lleven a los profesores a comprender la estrategia de la PR del profesional reflexivo como medio de la identificación del CPPB que construye el maestro en y sobre la acción.

La pregunta que orientará el desarrollo del taller será:

¿Cómo la reflexión sobre la práctica docente puede favorecer la construcción del conocimiento profesional del profesor de biología?

Primer principio -hacer consciente lo que hago y cómo lo hago- Tiene como propósito identificar que la práctica profesional que se lleva a cabo es producto del acontecer y del conocimiento que se ha construido con relación a la práctica pedagógica. Para esto se requiere un reconocimiento de lo que otros hacen para llegar a condusiones sobre lo que desde la práctica personal se lleva a cabo. Además, es necesario acercar al docente al reconocimiento, de algunos aspectos, de lo que él hace en el desarrollo de las tareas cotidianas, para así evidenciar la existencia de un saber que se produce gracias a su acción diaria. Es importante llevarlo a identificar cómo la acción que lleva a cabo en la práctica le permite construir un conocimiento que le es propio como profesor.

A continuación las preguntas orientadoras del trabajo que favorecerán el desarrollo del primer principio:

$>$ ¿Qué acciones he realizado desde mi práctica pedagógica?,

$>$ ¿Cuál es el conocimiento que se tiene cuando se está en la acción de la práctica?

$>$ Estando en la acción de la práctica ¿qué tipo de reflexiones se suscitan?

Segundo principio -reconocer el CPPB desde la reflexión sobre la práctica - Para esto es importante retomar algunas experiencias de los profesores en clase y desde allí, orientarlos a reconocer los procesos y las características ${ }^{2}$ de la acción desde los componentes del CPPB y desde ese planteamiento reflexionar acerca de las construcciones que se han elaborando producto de la práctica cotidiana en el aula. Así, al hacer énfasis en que al conocer la estructura propia del CPPB le permite al profesor construir un conocimiento producto de su quehacer diario y que en la medida que se siga trabajando sobre este conocimiento, esta actuación reflexiva le permitirá asentar las bases de su autodesarrollo profesional.

Para el desarrollo del segundo principio proponemos las siguientes preguntas orientadoras:

$>$ ¿Cuáles son las características que se quieren resaltar de la experiencia planteada?

$>$ ¿Por qué esas características?

$>$ ¿Qué resultados se obtuvieron?

$>$ ¿Por qué esos resultados y no otros?

$>$ ¿Qué cambios se dieron en el desarrollo de la práctica profesional a partir de la experiencia?

> A partir de la experiencia, ¿Cuál es la reinterpretación que logro hacer de mi práctica pedagógica?

${ }^{1}$ Según Pierre Bourdieu citado por Perrenoud (2007, p.13), se refiere al conjunto de los esquemas de que dispone una persona en un momento determinado de su vida. Por otro lado, define el habitus como un pequeño conjunto de esquemas que permite infinidad de prácticas adaptadas a situaciones siempre renovadas, sin constituirse jamás en un principio explícito.

${ }^{2}$ Los tópicos a tener en cuenta para el análisis de la experiencia son:

1. Las características de la situación que se considera.

2. Los objetivos propuestos para la realización de la experiencia establecida.

3. Los procedimientos que se llevaron a cabo.

4. El reconocimiento de las creencias y formas de representar la realidad vivida. 
Tercer principio -el hábitus de la Práctica Reflexiva como un fin en la construcción del CPPBHasta este momento en el taller los profesores habrán tenido que reconocer desde referentes experienciales puntuales las posibilidades de reflexión que se pueden llevar a cabo a través tanto de la reflexión en y sobre la acción, como elementos que favorecerán el reconocimiento del CPPB que se construye en el desarrollo profesional. Sin embargo para nosotros, la importancia del ejercicio está en invitar al desarrollo de una práctica constante como vía que le puede ofrecer al profesor una posibilidad de transformación personal, profesional que ha de partir de la práctica misma de una manera recurrente y sistemática. En otras palabras, la PR ha de ser una postura que se convierta en algo casi permanente y se inscriba dentro de una relación analítica-reconstructiva con la acción que se convierte en algo relativamente independiente de los obstáculos que aparecen o de las decepciones. Para lo anterior es definitivo, que el profesor genere altemativas que le permitan, a partir de su acción continuar con una PR veraz, continua y práctica ${ }^{3}$, esto se lograría a través del principio "Aprender a hacer lo que no se sabe hacer, haciéndold".

La construcción de la PR necesita de profesores capaces de decidir su propia forma de actuar, sus propios conocimientos, sus propios hábitus profesionales objetos de reflexión. Para esto consideramos que el reconocimiento del CPPB es fundamental, pues le permite afianzar y dar un estatus a lo que hace, no en función de lo que un profesor hace igual al otro, sino desde referentes que le son propios y producto de su experiencia.

Las preguntas que orientan el último principio son:

$>$ ¿Cómo hacer posible el desarrollo de la PR del CPPB sin que esto se convierta en una tarea más de trabajo?

$>$ ¿Cómo puede verse beneficiada la práctica profesional a partir del desarrollo de la PR?

Con el planteamiento de este taller no pretendemos brindar soluciones o recetarios que le permitan al profesor aualificar su labor. Para nosotros es más importante hacer énfasis, desde la experiencia del profesor, de la importancia que tiene pensar en el quehacer maestro como un campo de posibilidades, rica de ser creada y mejorada.

\section{Bibliografía}

Gutiérrez, A. (2008). Uso de estrategias metacogntivas y conocimiento profesional del profesor. Tesis de Maestría. Pontificia Universidad Javeriana. Bogotá.

Perrenoud P. (2007). Desarrollar la práctica reflexiva en el oficio de enseñar: Barcelona: Grao.

Porlán, R. Y Rivero, A. (1998). El conocimiento de los profesores: una propuesta formativa en el área de ciencias. Sevilla: Díada.

Schön, D. (1987). La formación de profesionales reflexivos. Hacia un nuevo diseño de la enseñanza aprendizaje de las profesiones. Barcelona: Paidos

Tardif, M (2004). Los saberes del docente y su desamollo profesional. Madrid: Narcea.

Valbuena, E. (2007). El Conocimiento Didáctico del Contenido Biológico. Estudio de las concepciones disciplinares y didácticas de futuros docentes de la Universidad Pedagógica Nacional (Colombia). Tesis de Doctor. Universidad Complutense de Madrid. Madrid.

${ }^{3}$ Cuando planteamos el término práctico nos referimos a que sea un ejercicio que se ajuste a la realidad del día a día del maestro.

${ }^{4}$ Planteado por Meinieu (1996) citado por Perrenoud (2007)

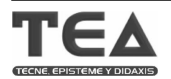

